\title{
SUPERSONIC MIXING AND COMBUSTION: ADVANCE IN LES MODELING
}

\author{
A. Ingenito and C. Bruno
}

Mixing and combustion in supersonic reacting flows are currently under investigation for new generation launchers and trans-atmospheric vehicles. Experimental results with hydrogen injected at Mach 2.5 in a Mach 2 airstreams showed combustion taking place just in $\sim 0.6 \mathrm{~m}$ : this indicates that supersonic combustion is feasible within short combustors. Large eddy numerical simulations including the subgrid scale (SGS) model, ISCM, developed specifically for supersonic combustion have been done. This model takes into account the effect of compressibility on reaction rates and on mixing. Numerical simulations have revealed that the flame is unsteady: it anchors at about $15 \mathrm{~cm}$ from the injector, develops downstream, and lifts off. Periodical ignition and quenching have been investigated. Also, the combustion regime in supersonic flows has been investigated and is reported.

\section{INTRODUCTION}

Large eddy simulations (LES) can be of support in designing supersonic combustor if a subgrid scale model suitable to supersonic flows is implemented. In fact, numerical simulations with the Smagorinsky-Lilly SGS model often predict neither mixing nor combustion whilst experiments show both occurring over short lengths. Past evidence [1] shows that the physics of mixing and combustion is very different in subsonic and supersonic regimes. From a previous theoretical analysis [2] of the effects of high Mach number on turbulence and combustion, it has been observed that high Mach number flows experience mainly streamwise vorticity and consequently maximum helicity. Both affect mixing and improve it at large scales. It has also been found that mixing can be improved by forcing transversal pressure gradients, e.g., by means of particular geometries (generating shocks) and devices (injectors).

In this test case, the bow shock due to the transversal injection induces the vorticity. Furthermore, it has been shown that supersonic combustion takes place locally at approximately constant volume, and that collisional frequency increases due to local dilatation $[3,4]$ resulting in faster kinetics. Based on 
the previous observations, a novel SGS model, ISCM, has been developed. The ISCM model accounts for compressibility effects on mixing and on combustion. In particular, microscale physics has been included by means of a subgrid kinetic energy equation that is algebraically modeled to provide the velocity fluctuation needed by the eddy viscosity SGS closure. The SGS model (ISCM) accounts convincingly for key physics of supersonic combustion and can be used to suggest how to improve the supersonic combustor design.

\section{LARGE EDDY SIMULATIONS OF SUPERSONIC COMBUSTION WITH THE ISCM MODEL}

\subsection{NASA-Langley Test Case Geometry and Experimental Results}

The geometry of the scramjet (SCRJ) combustor configuration is shown in Fig. 1. A direct-connect supersonic combustor model, known by its acronym SCHOLAR has been developed at NASA-Langley Research Center. This configuration is that used during the NATO ATV-10 RTO project a few years ago [5]. This experiment has been designed to provide optical access to a reacting supersonic flowfield typical of the flow present in a scramjet engine.

The model shown in Fig. 1 consists of a section $546 \mathrm{~mm}$ in length made of copper for thermal control followed by a 914-millimeter long section made of carbon steel attached to the aft end of the copper section. The copper section contains a single fuel injector that introduces gaseous hydrogen into the vitiated air stream flowing through the model.

The injector region of the combustor model is shown in Fig. 1b. The model consists of a constant area channel initially $38.6 \mathrm{~mm}$ high and $87.9 \mathrm{~mm}$ wide followed by a 4.8-millimeter rearward-facing step and a 43.8-millimeter long constant area section. Combustion heated vitiated air is introduced into the channel at Mach 2, $1184 \mathrm{~K}$, and 100,405 $\mathrm{Pa}$.

The vitiated air contains 20.35 percent water by mass introduced by the facility heater. The injector lies on the duct centerline. The fuel injector is inclined at $30^{\circ}$ to the horizontal and has a circular cross-section $7.6 \mathrm{~mm}$ in diameter. It is located just downstream of the $3^{\circ}$ divergence $(\sim 43.8 \mathrm{~mm}$ from the step) section. A 3-degree expansion of the upper wall begins immediately at the primary fuel injector. This 3-degree expansion continues along the upper wall of the carbon steel section that is attached to the copper combustor model.

Five measurement locations for optical access are provided in the copper part of the combustor model. Two additional measurement stations are provided in the carbon steel section. From these single-shot measurements, averages and root 


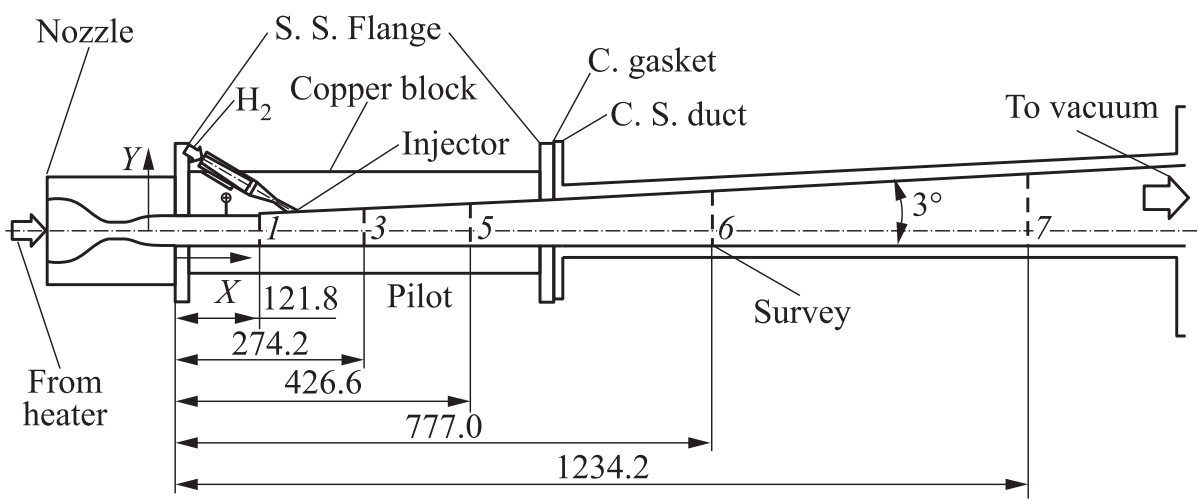

(a)

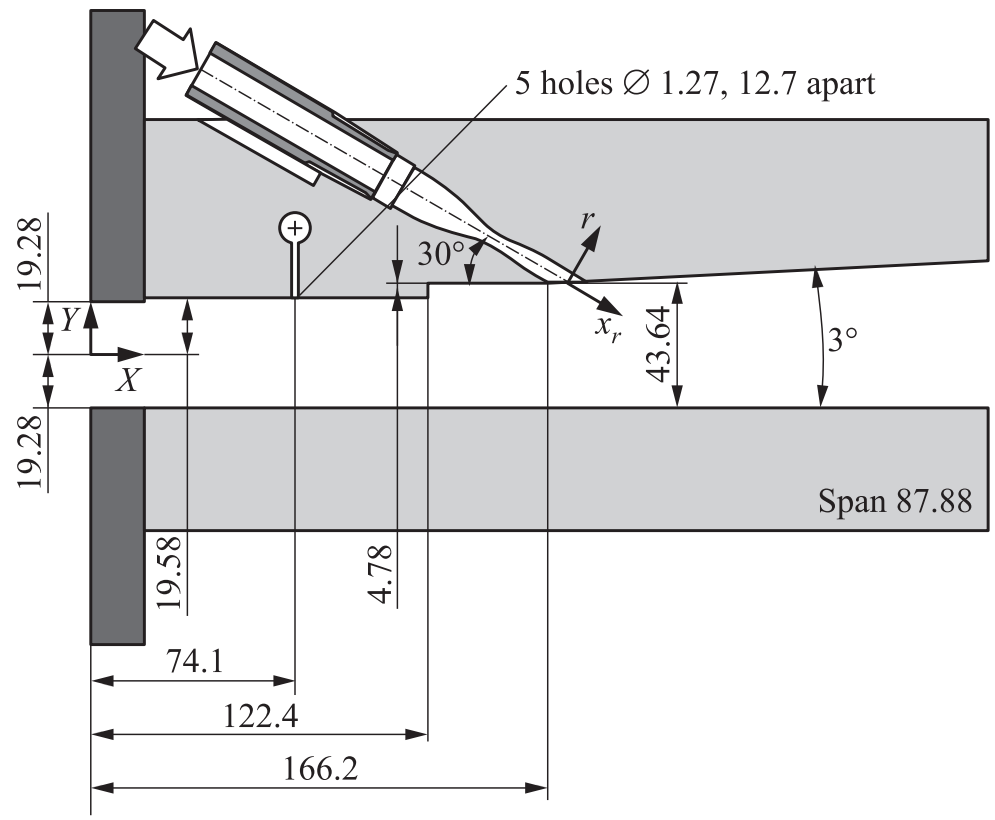

(b)

Figure 1 Test model: (a) nozzle and combustion chamber and $(b)$ detail in the vicinity of fuel injector. Dimensions are in millimeters 


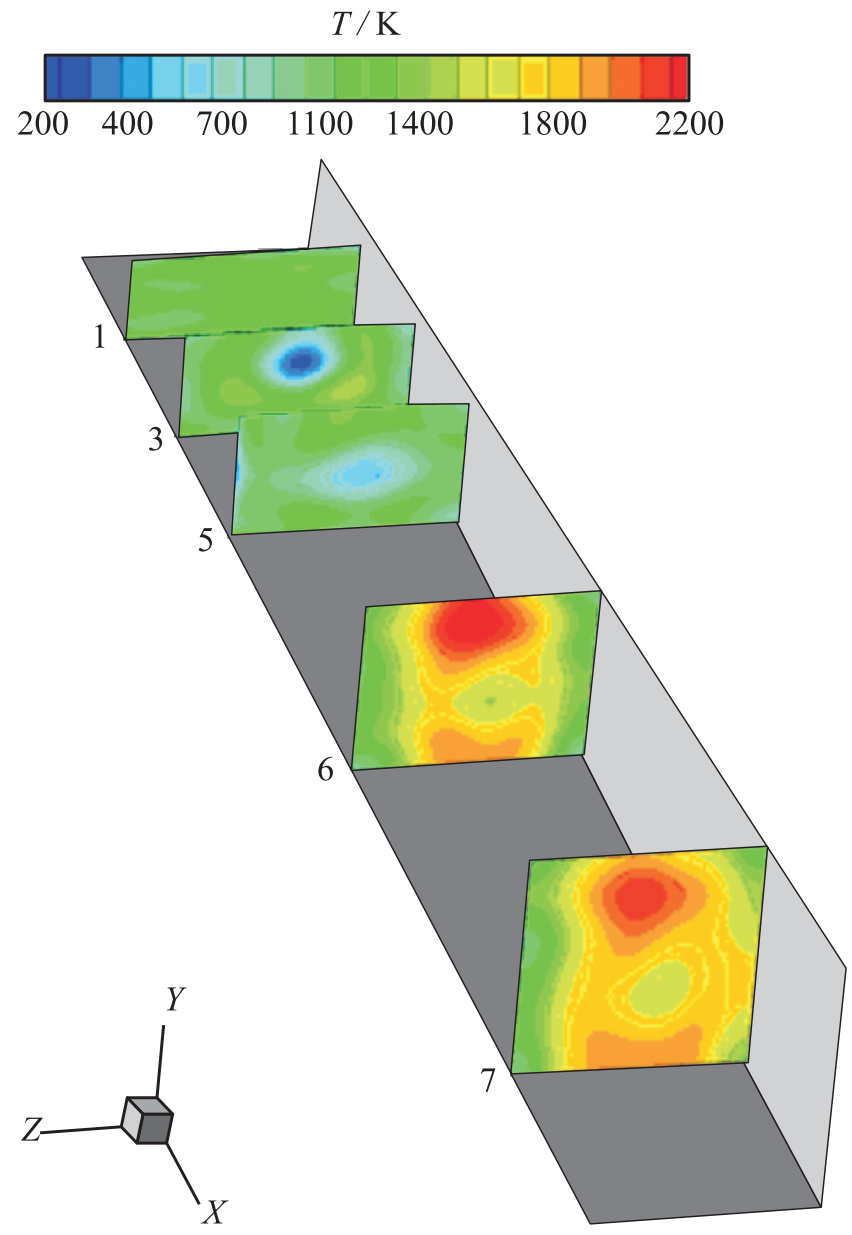

Figure 2 Three-dimensional view of the experimental average temperature field. (Refer Ingenitoand Bruno, p. 518.)

Table 1 Vitiated air mole fractions at the inlet

\begin{tabular}{lc}
\hline Species & Mole fraction \\
\hline $\mathrm{H}_{2}$ & $1.3 \cdot 10^{-5}$ \\
$\mathrm{OH}$ & $7.1 \cdot 10^{-4}$ \\
$\mathrm{O}_{2}$ & 0.21 \\
$\mathrm{H}_{2} \mathrm{O}$ & 0.217 \\
$\mathrm{~N}_{2}$ & 0.5722 \\
\hline
\end{tabular}

mean square (RMS) values are derived. In addition to optical measurements, wall pressures are measured using an array of orifices.

Figure 2 shows a three-dimensional (3D) view of the experimental average temperature field. Experimental results predict the flame anchoring between cross sections 5 and 6. As this last section is at $77 \mathrm{~cm}$ from the combustor inlet plane and at about $60 \mathrm{~cm}$ from the $\mathrm{H}_{2}$ injector, mixing times can be es- 
timated to be $\sim 5 \cdot 10^{-4} \mathrm{~s}$ (Table 1 ). This means that this supersonic flow mixes and burns in a very reasonably short time. Figure 2 also shows that the flame is developed both at the bottom and upper wall. Maximum (averaged) temperatures are about 2200 to $2300 \mathrm{~K}$.

\subsection{Numerical Scheme}

Three-dimensional LES were performed with FLUENT $6.3^{\mathrm{TM}}$ commercial code [6] to simulate the flowfield in the NASA-Langley combustor model. Double precision has been used.

The LES solver is a coupled, upwind, explicit, third-order MUSCL*, accurate both in space and time. This scheme was conceived from the original by blending a central differencing scheme and second-order upwind scheme. In FLUENT, compared to the second-order upwind scheme, the third-order MUSCL has the potential to improve spatial accuracy for all types of meshes by reducing numerical diffusion, most significantly for complex 3D flows.

\subsection{Computational Domain}

The SCRJ combustor geometry of Fig. 1 has been mapped by a 3D grid (Fig. 3).

This domain was discretized with a grid of 700 points in the streamwise direction, 46 points in the cross-stream direction, and 60 points in the spanwise direction. The grid was made finer near the step and the fuel injector. The number of hexahedral cells of the computational domain is $1,563,994$, for a total of $1,626,578$ nodes. Only the first $800 \mathrm{~mm}$ of the whole length of the combustor $(1234.2 \mathrm{~mm})$ have been simulated. With this grid, a very small wall $Y^{+}$, of order of $4-8$ has been obtained near the step and the $\mathrm{H}_{2}$ injector, where the NASA experiment predicts bow shock formation (Fig. $3 a$ ). At the inlet, instead, the grid should be further refined to allow a better prediction of the boundary layer (Fig. $3 b)$.

However, both the shock departing from the step and that just upstream the fuel injection are predicted by simulations, this leading the two counter rotating vortices generation along the stream direction. Also shocks reflection between the upper and the bottom walls is predicted.

\subsection{Inlet Boundary Conditions}

Calculations begin at the $x=0$ station of the SCHOLAR model, where vitiated air from the facility enters the duct. The initial channel cross-section is $38.6 \mathrm{~mm}$

\footnotetext{
${ }^{*}$ MUSCL - Monotone Upstream-centered Schemes for Conservation Laws.
} 

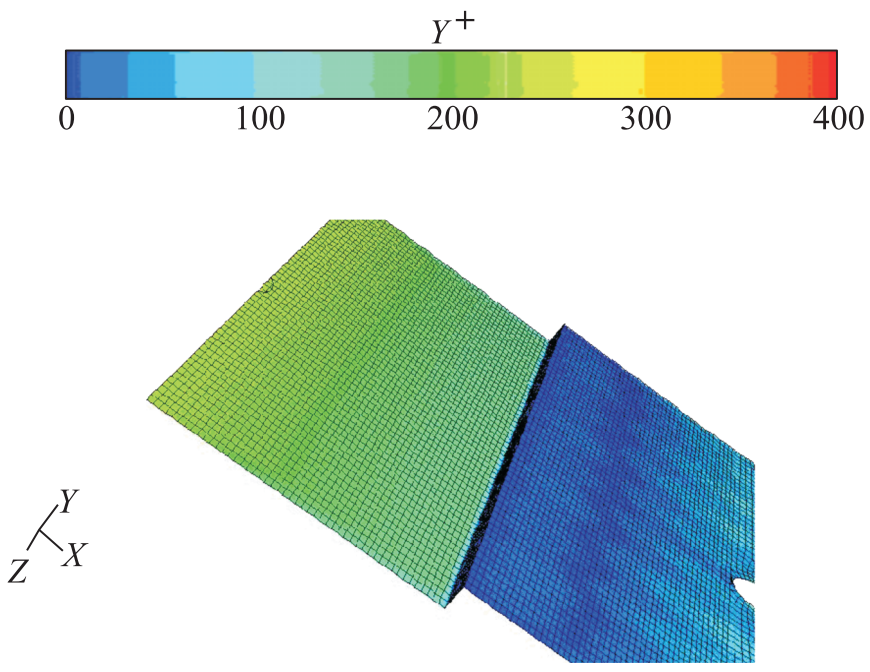

(a)

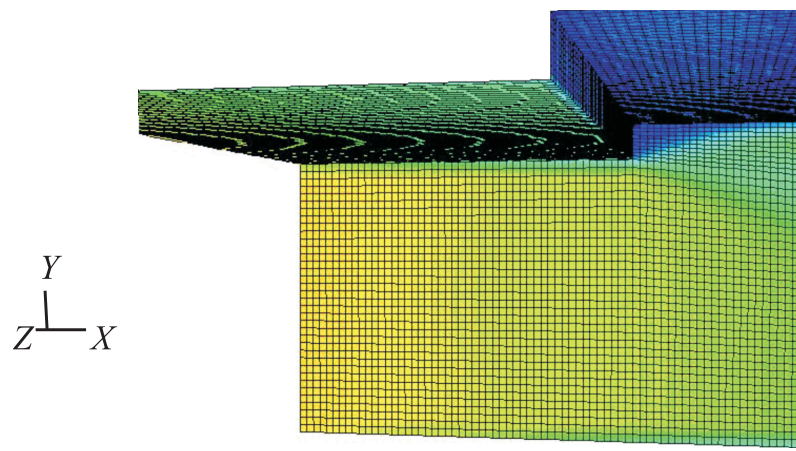

(b)

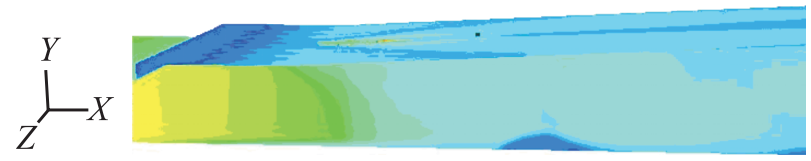

(c)

Figure 3 Instantaneous contours of wall $Y^{+}$in the first part of the combustor $(a)$; at the combustor inlet upper wall $(b)$; and in the second part of the combustor $(c)$. (Refer Ingenito and Bruno, p. 520.) 
high and $87.9 \mathrm{~mm}$ wide. Vitiated air enters the model at Mach 2.0 yielding a velocity of $1395.7 \mathrm{~m} / \mathrm{s}$, a static temperature of $1204 \mathrm{~K}$, and a static pressure of $101,325 \mathrm{~Pa}$. Air inlet temperature is sufficiently high to produce self-ignition of the air-hydrogen mixture.

The mole fractions of the species present in the vitiated air are given in Table 1.

The hydrogen fuel injector introduces hydrogen at Mach 2.5 with a static temperature of $1343 \mathrm{~K}$, and a static pressure of $202,650 \mathrm{~Pa}$. The air $/ \mathrm{H}_{2}$ equivalence ratio is $\sim 1$.

Combustor walls are nonadiabatic; in fact, the measured heat flux varies linearly from 0.7 to $1.8 \mathrm{MW} / \mathrm{m}^{2}$ along the $x$ direction, corresponding to about $10 \%$ of the combustion heat release. The heat flux function has been implemented as a boundary condition for the energy equation by means of a user defined function in the FLUENT code used in these simulations.

No-slip conditions were specified along the channel walls.

\subsection{Turbulence and Chemistry Modeling}

The SGS model used for the turbulent closure of the filtered Navier-Stokes equations is the ISCM model [4]. In brief, in the ISCM model:

(i) microscale physics has been included by means of a subgrid kinetic energy equation that is algebraically modeled to provide the velocity fluctuation to be used in an eddy viscosity SGS closure;

(ii) reacting turbulent structures have been modeled by means of a reactor burning at constant volume; and

(iii) the reaction rate depends on the local Mach number.

Hydrogen/air chemical kinetics has been modeled by means of an overall 1-step reaction involving 3 reacting species [7]:

$$
\mathrm{H}_{2}+0.5 \mathrm{O}_{2}=\mathrm{H}_{2} \mathrm{O} \quad k_{\mathrm{ov}}=1.8 \cdot 10^{13} \exp \left(-\frac{17614}{T}\right)\left[\mathrm{H}_{2}\right]^{1.0}\left[\mathrm{O}_{2}\right]^{0.5} .
$$

This scheme does not consider the effects of the small quantities of radicals present in the vitiated air. However, the assumption of 1-step mechanism instead of a more detailed mechanism can be justified considering that anchoring at this air temperature and pressure is not controlled by kinetics.

In fact, the results obtained by CHEMKIN 3.7 code, concerning hydrogen/air combustion performed with a detailed mechanism consisting of 9 species and 20 reactions, have shown (for the same NASA-Langley test case conditions) very short ignition delay times, of order of $0.05 \mathrm{~ms}$, ten times lower than the 
mixing time, as estimated from the experiments. Besides, comparing the results obtained by CHEMKIN 3.7 code for the same NASA-Langley test case conditions with a 1 step and a more detailed $\mathrm{H}_{2}$ /air mechanism, a negligible difference in terms of ignition delay time and flame temperature is shown.

\subsection{Simulations}

Simulations performed with the ISCM subgrid model show the $\mathrm{H}_{2}$ jet penetrating up to 0.5 diameter, and then bending down towards the bottom wall. A bow shock is located immediately in front of the injector (Fig. 4). Shock reflections between upper and bottom walls are predicted. As is seen from Fig. 4, vorticity is mainly streamwise.

Figure 5 shows averaged temperature fields at sections 5 and 6 . Two counterrotating vortices move along the stream direction, spread the $\mathrm{H}_{2}$ jet enhancing fuel-air mixing and reaction. The vortices also convect fluid toward the lower wall. Experimental data indicate a maximum temperature of between 2030 and $2300 \mathrm{~K}$, while simulations predict the maximum (averaged) temperature of $2400 \mathrm{~K}$. A "cold" core of unreacted hydrogen persists in both experimental data and calculation at the end of combustor.

Numerical simulations show also unsteady flame behavior similar to what is reported in [8]. In the numerical simulations, combustion starts on the upper side of the hydrogen-air shear layer, at about $5 \mathrm{~cm}$ from the $\mathrm{H}_{2}$ injector (Point 1 in Figs. 4 and 6). Here, the mixture ignites periodically at about $2500 \mathrm{~Hz}$. Mixture ignites also at about $15 \mathrm{~cm}$ from the $\mathrm{H}_{2}$ injector (Point 2 in Figs. 4 and 6). From this latter point, the flame develops downstream, lifts off and is convected towards the exit. This phenomenon is periodical and forced by the ignition of reactants at point 1 . After $0.4 \mathrm{~ms}$, the flame starts again to develop downstream
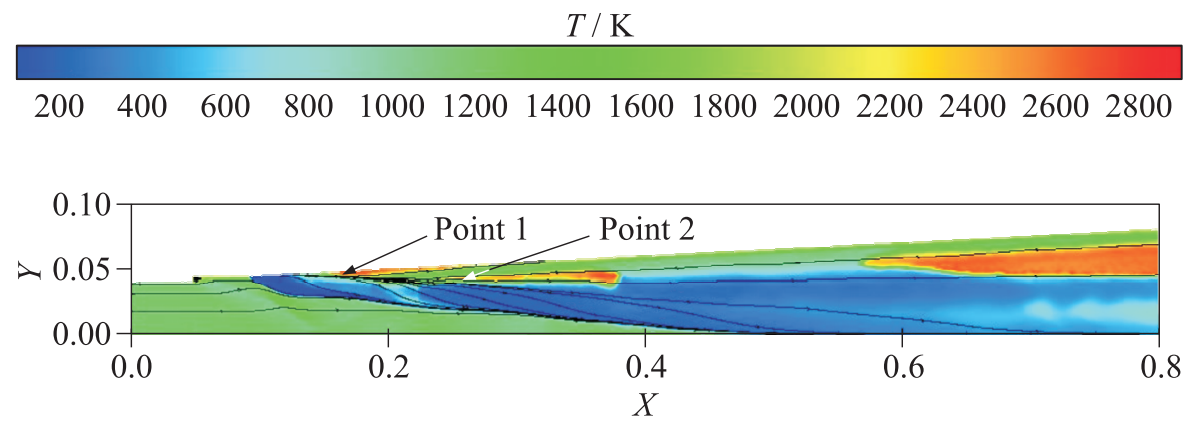

Figure 4 Instantaneous temperature field and streamlines in the middle $X Y$ plane. (Refer Ingenitoand Bruno, p. 522.) 


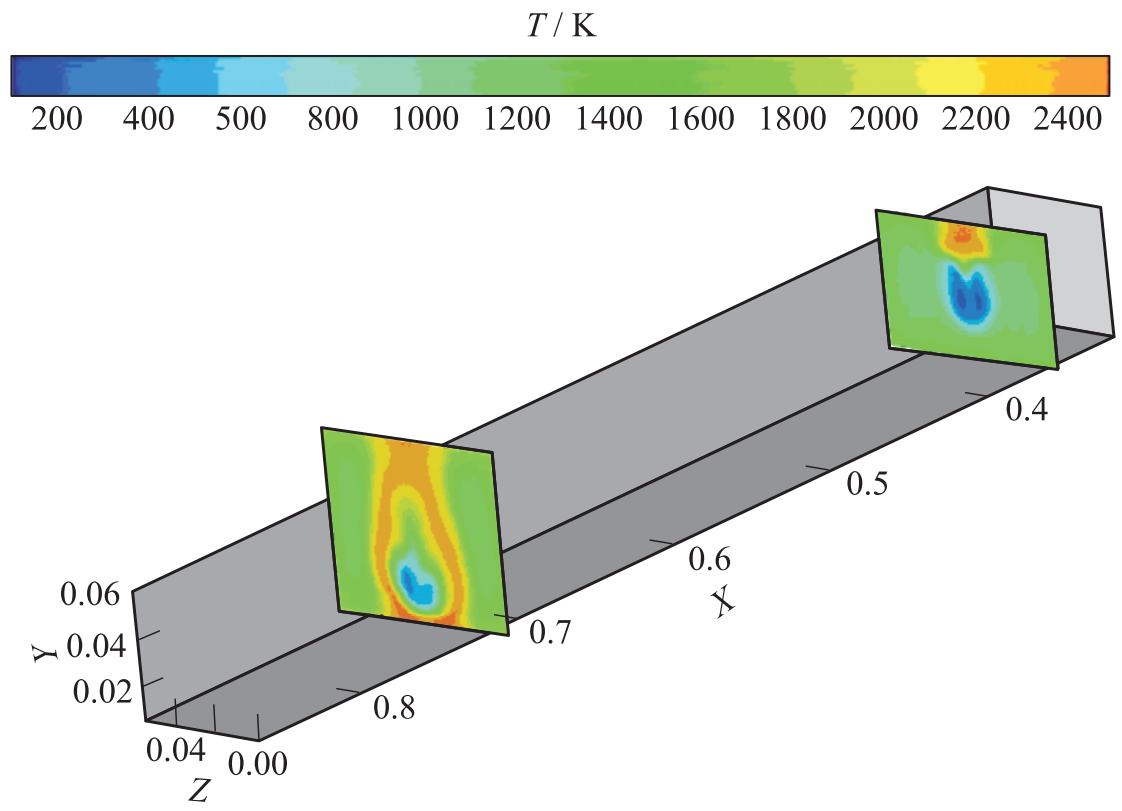

Figure 5 Three-dimensional view of the numerical averaged temperature field at $X=0.3$ and $0.7 \mathrm{~cm}$. (Refer Ingenito and Bruno, p. 523.)
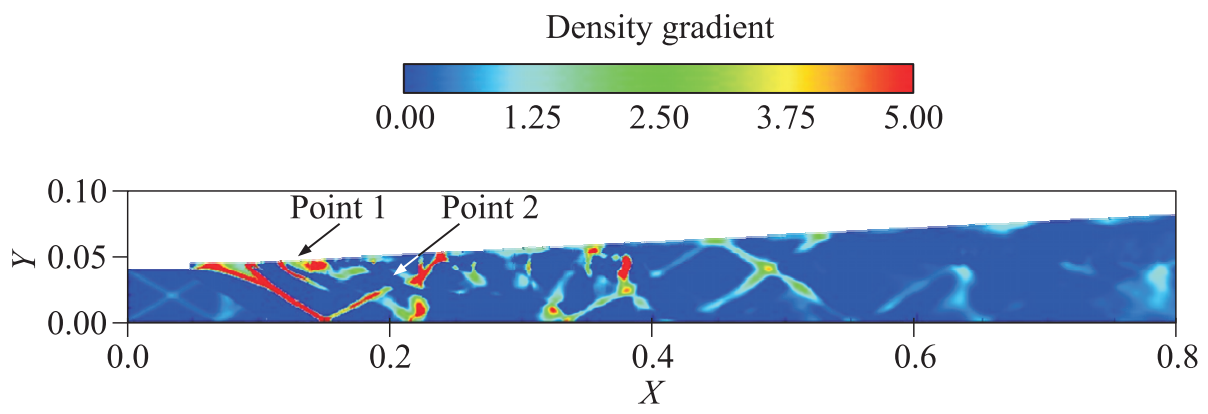

Figure 6 Visualization of shock waves, ISCM model. (Refer Ingenito and Bruno, p. 523.)

and the whole phenomenon repeats. This means that the local temperature and equivalence ratio allow mixture reignition. It follows from Fig. 4 that instantaneous maximum temperatures are higher than temperatures measured in the experiments by $15 \%-20 \%$. This is likely due to the use of a single-step oxidation reaction with no radicals in the products. The two counterrotating vortices ex- 
isting in all cross sections promote mixing, thereby favouring flame propagation, albeit unsteady.

Combustion at the bottom of the combustor starts between sections 5 and 6 . The flame length, in good agreement with the experimental results, reaches the end of combustor and continues out.

According to Figs. 4 and 6, flame anchors on the upper wall, at $5 \mathrm{~cm}$ from the injector, and occurs in correspondence to the shock wave, where the density gradient is higher. This means that shocks play an important role in flame anchoring, possibly due to increased mixing rates caused by the coupled effect of the density and pressure gradients on vorticity transport (baroclinic effect), and also due to the influence of the dilatation term on the reaction rate. Numerical results show that shock waves are steady; therefore, the pulsating behavior of the flame is not due to shock oscillations.

Numerical results point out that peaks of subgrid velocity fluctuations, defined here as $\sqrt{k_{\mathrm{SGS}}}$, are less than $20 \mathrm{~m} / \mathrm{s}$ and that turbulent viscosity predicted by the ISCM model is smaller than $0.001 \mathrm{~kg} /(\mathrm{m} \cdot \mathrm{s})$. Maximum values are shown at the hydrogen/air interface and in the locations where combustion occurs. Mixing and "instabilities" are analyzed in the following sections.

\section{MIXING AND COMBUSTION FOR SUPERSONIC FLOWS}

Flame-holding and mixing are the critical issues in the design of supersonic/ hypersonic combustors. In fact, in supersonic flows, the residence time of the air stream in the combustion chamber is very short: for an airstream entering at $\mathrm{Ma}=2$ in a combustor $1 \mathrm{~m}$ long, it is about a millisecond. In this short time, fuel and air must mix, ignite, and burn. Incomplete or nonuniform mixing leads to unburned reactants with resulting loss in efficiency and may lead to production of pollutants.

In principle, supersonic combustion is so fast that $\mathrm{NO}_{x}$ production may be considerably less than in conventional subsonic combustion. The ideal case of complete mixing is extremely difficult to achieve in practice. Increasing combustor length is not advisable due to skin friction losses: in fact, in a SCRJ vehicle, the combustor is responsible for about $60 \%$ of the total skin friction drag [9]. To improve mixing without increasing the combustor length, several solutions have been investigated: transverse injection, lobe mixers [10], vortex generators, and by devices exploiting the interaction with shock waves. The major drawback is in terms of thrust penalty: in fact, thrust losses scale with increasing jet Mach number [11].

Transverse injection, as in the NASA-Langley test case, offers relatively rapid mixing and good fuel penetration. In fact, penetration of the fuel stream into 
the cross-flow is governed by the jet-to-freestream momentum flux ratio. The fuel jet interacts strongly with the cross-flow, producing a bow shock and a localized highly $3 \mathrm{D}$ flow field. Vorticity addition to the air stream provides more significant mixing enhancement of fuel and air.

In order to estimate ignition times, the NASA-Langley test case initial conditions have been assumed, in particular: $V=1200 \mathrm{~m} / \mathrm{s}, P=1 \mathrm{~atm}$, and $\Phi=1$. The averaged mixture temperature (between air at 1204 and fuel at $103 \mathrm{~K}$ ) is therefore about $1180 \mathrm{~K}$. The chemical time, $\tau_{\text {chem }}$, for a well mixed reactor, is about $\sim 50 \mu \mathrm{s}$.

The distance at which spontaneous ignition occurs in a medium flowing at a velocity $U$ is of order $L_{\text {chem }}=U t_{i} \sim 6 \mathrm{~cm}$. This distance is in good agreement with that predicted by numerical simulations $(\sim 5 \mathrm{~cm})$.

Because of these small ignition delay times, the Damköhler number, defined as the ratio between characteristic convective and chemical times, is:

$$
\operatorname{Da}_{I}=\frac{\tau_{\text {conv }}}{\tau_{\text {chem }}} \cong \frac{833}{50} \cong 16>1
$$

This means that if fuel and air are well mixed, ignition occurs within the combustor, and as a consequence chemistry does not control combustion.

Once established that flame anchors, a study on the reasons for flame unsteadiness has been done. In fact, supersonic flows where large temperature changes occur across the shocks, at the hydrogen-air interface, and across the flame, the reaction rate $\omega_{r}=\exp \left(-E /\left(R T_{i}\right)\right)\left(E\right.$ is the activation energy and $T_{i}$ is the initial reactant temperature) may change by orders of magnitude. Since the activation energy is generally large (here is of order $35 \mathrm{kcal} / \mathrm{mol}$ ), the reaction rate is very temperature-sensitive. Thus, any fluctuation in the flame temperature will result in a large change in the reaction rate, leading to 'instability' of the flame front. Furthermore, rapid density changes across the flame can drive acoustic wave generation that can couple with the increase of the burning rate to induce acoustic-driven instability. Combustion unsteadiness is likely due to this interaction between heat release, acoustics, and kinetics.

In order to understand this periodical behavior, an analysis of the characteristic times has been performed. In fact, because of the known $S$-curve for hydrogen/air autoignition, if the temperature of the mixture is below a threshold temperature of about 900-1000 K, kinetics becomes slower. For a SCRJ combustor, where reactants are not premixed but, for instance, cold hydrogen is injected into hot air, temperature varies significantly with the equivalence ratio throughout the mixing layer around the jet. At lower temperature, ignition delay times decrease and combustion become eventually controlled by kinetics [12]. As shown in the previous section, the ignition delay time $(\sim 0.05 \mathrm{~ms})$ is smaller than the residence time $\left(\tau_{\text {conv }} \sim L / U=0.83 \mathrm{~ms}\right)$, so kinetics is fast enough to let the flame ignite during a convective time. 

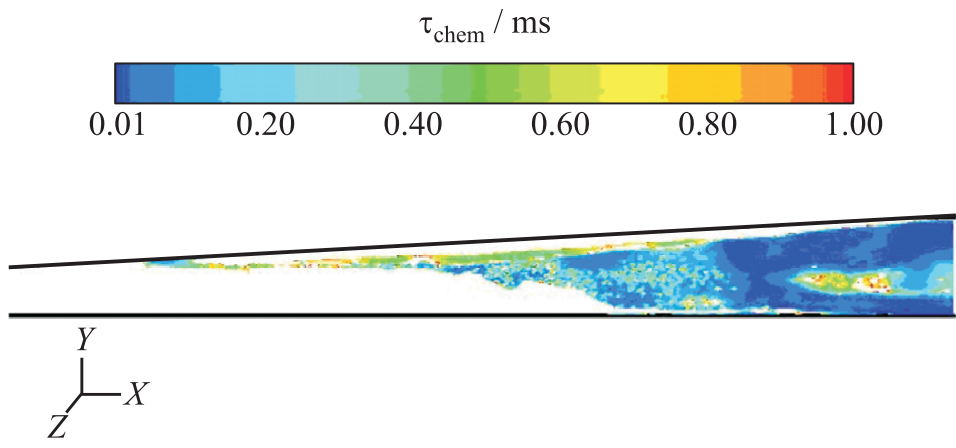

Figure 7 Instantaneous contour of the chemical time. (Refer Ingenito and Bruno, p. 526.)

An analysis of mixing at small scales has been conducted by looking at the local value of Da, M, and Re numbers. By looking at the instantaneous field of chemical times (Fig. 7), it turns out that in the part of combustor downstream of the injector, these times are shorter than $1 \mathrm{~ms}$. In particular, the chemical times, calculated by:

$$
\tau_{\text {chem }}=\left(\frac{\dot{\omega}}{\rho}\right)^{-1}
$$

vary from 0.01 to $1 \mathrm{~ms}$ in the zone where combustion takes place.

Turbulent convective times in the flow field, calculated as a ratio between the cell dimension and the SGS velocity fluctuations:

$$
\tau_{\text {conv }}=\frac{\Delta}{\sqrt{k_{\mathrm{SGS}}}}
$$

range from about 0.8 to $1 \mathrm{~ms}$ (Fig. 8).

The local turbulent $\mathrm{Da}_{t}$ number, i.e., the ratio of the turbulent convective time to the chemical time, calculated in each computational cell is shown in Fig. 9. In the regions where $\mathrm{Da}_{t}<1$, combustion does not occur. The values of $\mathrm{Da}_{t}>1$ are present only in the second part of the combustor, downstream of the injector. The maximum $\mathrm{Da}_{t}$ values are of order of 90 , even if the $\mathrm{Da}_{t}$ number assumes generally values of about $20-40$.

Figure 10 shows that in the locations where combustion occurs, the Mach number decreases. This is due to the effect of heat addition to a supersonic flow in a constant area combustor, as predicted by Rayleigh. In this case, due to the fact that the chamber is slightly divergent, the flow stays supersonic and does not choke.

Damköhler $\left(\mathrm{Da}_{t}\right)$ and Reynolds $\left(\mathrm{Re}_{t}\right)$ numbers predicted by numerical simulations are in the same regime of that predicted by the Williams-Klimov diagram 


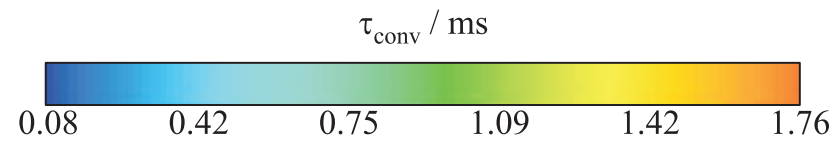<smiles>[Y][C][Y]</smiles>

Figure 8 Instantaneous contour of the turbulence convective time. (Refer Ingenito and Bruno, p. 527.)
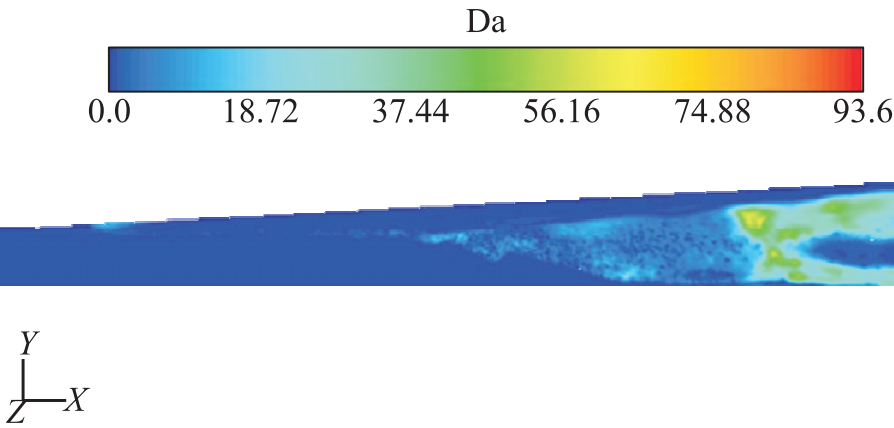

Figure 9 Instantaneous contour of the Damkohler number. (Refer Ingenito and Bruno, p. 527.)

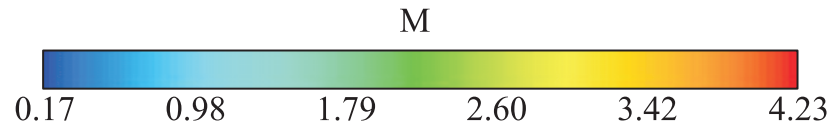

$$
\stackrel{Y}{Z}^{Y} X
$$

Figure 10 Instantaneous contour of the Mach number. (Refer Ingenito and Bruno, p. 527.) 


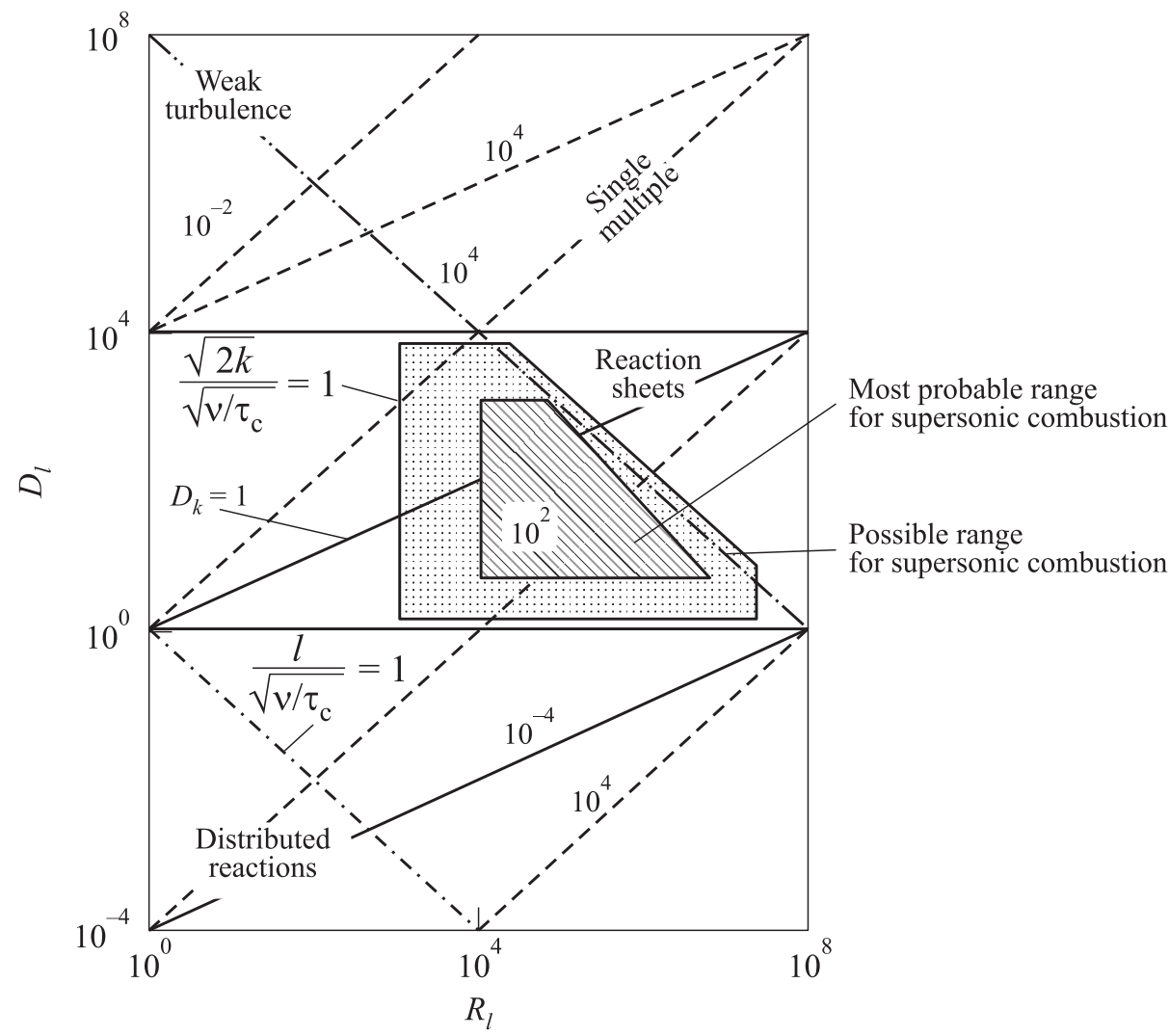

Figure 11 Williams-Klimov diagram

for subsonic flames (Fig. 11). The combustion regime for these characteristic numbers corresponds to the reaction sheet regime: this means that the flame is corrugated.

\section{CONCLUDING REMARKS}

In this work, numerical simulations of the NASA-Langley test case have been performed. These simulations have pointed out that the flame is unsteady: it anchors and quenches periodically. This may be explained as due to the effect of the high temperature gradients caused by shock waves and local compression on the reaction rate. In fact, below a threshold temperature of about 900-1000 K, ignition delay times may become longer than the mixing times. 
By a comparison with the large-scale characteristic times in a supersonic combustor, it can be shown that the chemical time is shorter than the residence time and the flame anchors within the combustor.

By comparing small-scale characteristic times, one may tentatively conclude that combustion occurs in the reaction sheet regime.

\section{ACKNOWLEDGMENTS}

This work was performed within the 'Long-Term Advanced Propulsion Concepts and Technologies' (LAPCAT) project investigating high-speed air-breathing propulsion. The LAPCAT project, coordinated by ESA-ESTEC, is supported by the EU within the 6th Framework Programme Priority 1.4, Aeronautic and Space, Contract No. AST4-CT-2005-012282. Further info on LAPCAT can be found on http://www.estec.esa.int/techresources/lapcat.

\section{REFERENCES}

1. Ingenito, A., M. G. De Flora, E. Giacomazzi, C. Bruno, and J. Steelant. 2006. LES modeling of scramjet combustion. 44th AIAA Aerospace Sciences Meeting and Exhibit. Reno, NV.

2. Ingenito, A. 2005. Supersonic combustion modeling. Ph.D. Dissertation. Mechanics and Aeronautics Department, University of Rome "La Sapienza," Rome. (In Italian.)

3. Giordano, D. 2003. The influence of medium compressibility on chemical-reaction rates. Part I: Theoretical considerations. AIAA Paper No. 2003-4057.

4. Ingenito, A., M. G. De Flora, E. Giacomazzi, C. Bruno, and J. Steelant. 2006. A novel model of turbulent supersonic combustion: Development and validation. 42nd AIAA/ASME/SAE/ASEE Joint Propulsion Conference and Exhibit. Sacramento, CA.

5. Drummond, J. P., G.S. Diskin, and A. D. Cutler. 2001. Fuel-air mixing and combustion in scramjets. Technologies for propelled hypersonic flight. NATO Research and Technology Organization, Working Group AVT 10. Final Report.

6. http://www.fluent.com.

7. Marinov, N. M., C. K. Westbrook, and W. J. Pitz. 1996. Detailed and global chemical kinetics model for hydrogen. In: Transport phenomena in combustion. Vol. 1. Washington, DC: Taylor and Francis. 118-29.

8. Choi, J., and V. Yang. 2003. Flow oscillations in hydrogen-fueled scramjet combustors. International Colloquium on Hypersonic Propulsion. Beijing, China.

9. Paull, A., R. J. Stalker, and D. J. Mee. 1995. Experiments on supersonic combustion ramjet propulsion in a shock tunnel. J. Fluid Mech. 296:159-83. 
10. Campuzano, M.F., and T. Q. Dang. 1995. Numerical study of lobed-mixer fuelinjection strut in scramjet engine. 31st ASME, SAE, and ASEE, Joint Propulsion Conference and Exhibit. San Diego, CA.

11. Stalker, R. J., A. Paull, D. J. Mee, R. G. Morgan, and P. A. Jacobs. 2005. Scramjets and shock tunnels - the Queensland experience. Prog. Aerospace Sci. 41(6):471513.

12. Ingenito, A., E. Giacomazzi, C. Bruno, and J. Steelant. 2006. LES modeling of scramjet combustion. 14th AIAA/AHI Space Planes and Hypersonic Systems and Technologies Conference. Canberra, Australia. 